\title{
Reversal of Left Ventricular Function by PVC Ablation in Dilated Cardiomyopathy Patient
}

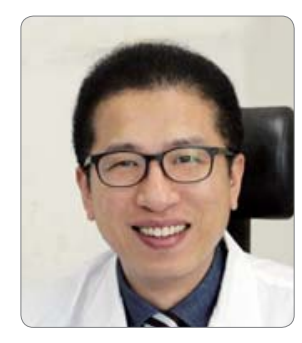

Seung Jin Jun

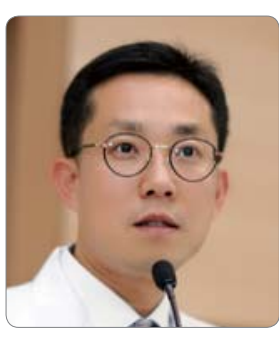

Ki Hong Lee
Seung Jin Jun, MD1; Ki Hong Lee, $\mathrm{MD}, \mathrm{PhD}^{2}$

${ }^{1}$ Division of Cardiology, Gunsan Medical Center, Gunsan, Korea

${ }^{2}$ Division of Cardiology, Chonnam National University Hospital, Gwangju, Korea
Received: February 9, 2018

Accepted: March 22, 2018

Correspondence: Ki Hong Lee, MD, PhD, FESC

Associate Professor, Cardiovascular Medicine,

The Heart Center of Chonnam National University

Hospital, 42 Jaebongro, Dong-gu, Gwangju 501

757 , South Korea

Tel: +82-62-220-6246, Fax: +82-62-223-3105

E-mail: drgood2@naver.com

Copyright (c) 2018 The Official Journal of Korean Heart Rhythm Society Editorial Board

\begin{abstract}
Premature ventricular complex (PVC) usually follows a benign course and shows good response to medical therapy. However, high burden of PVC deteriorates cardiac function and is often associated with progression into dilated cardiomyopathy (DCMP). We report a case of a young patient who recovered from DCMP after PVC ablation. The patient complained of palpitations and dyspnea on exertion. Holter examination revealed an isolated PVC burden of $29 \%$. Despite intensive medical therapy for more than a year, symptoms aggravated and PVC burden was not diminished on followup Holter examination. Furthermore, the echocardiogram revealed deteriorated systolic function as well as left ventricular enlargement, indicating progression into DCMP. Surface electrocardiogram indicated PVC origin in the left ventricular outflow tract. Detailed mapping at the right ventricle and left ventricle outflow tract with the aid of 3-dimensional mapping system, demonstrated PVC origin from the left ventricular outflow tract area, between the right and left coronary cusps. Radiofrequency ablation successfully abolished all ventricular premature beats. Follow-up Holter examination revealed no PVC, and the echocardiogram showed recovery to normal systolic function and chamber size. In conclusion, ablation of PVC should be considered when it does not respond to medical therapy and is associated with deterioration of cardiac function.
\end{abstract}

Key Words: - Ventricular Premature Complexes

- Dilated Cardiomyopathy - Catheter Ablation

\section{Introduction}

Symptomatic premature ventricular complex (PVC) in structurally normal hearts usually takes a benign course and responds well to medical treatment including beta-blockers, and class Ic anti-arrhythmic drugs. However, not all patients respond 
to medical treatment, and sometimes progress to dilated cardiomyopathy (DCMP) due to high burden of PVC. ${ }^{1,2}$ In that case, class Ic anti-arrhythmics should be changed to amiodarone to avoid lethal ventricular arrhythmias. If all available antiarrhythmic agents fail to suppress PVC and systolic function declines, catheter ablation might be a good option to manage PVC and restore normal systolic function and chamber size. ${ }^{3}$ We report a case of a young male patient who was refractory to 4 different classes of anti-arrhythmic drugs, and progressed to PVCinduced DCMP, but was successfully treated and normal systolic function and chamber size were restored, without any remnant symptoms, by catheter ablation.

\section{Case}

An 18-year-old male patient presented with palpitations and dyspnea on exertion. Electrocardiogram (ECG) showed normal sinus rhythm and frequent isolated premature ventricular complex (PVC), whose origin was suspected to be in the left ventricle outflow tract (LVOT) right coronary cusp (RCC) (Figure 1). Holter examination demonstrated total PVC burden as $29 \%$.
Echocardiogram revealed normal systolic function and chamber size. Beta blockers were selected for initial management. However, symptoms were not relieved, with frequent isolated PVC still seen on a 12-lead ECG. Then, flecainide, propafenone were serially added, to reduce the symptoms and PVC burden. After one year of medication, follow-up echocardiogram demonstrated decreased systolic function of about $40 \%$ by the modified Simpson method, and dilated left ventricular end-diastolic dimension of $66 \mathrm{~mm}$, which indicated progression into dilated cardiomyopathy (DCMP) (Figure 2 A, B). Class Ic antiarrhythmic drugs were changed to amiodarone due to development of systolic heart failure. Nonetheless, total burden of PVC was not diminished on Holter examination. Dyspnea and chest discomfort gradually aggravated during medical treatment. Because PVC was refractory to medical treatment, radiofrequency ablation of PVC was planned. Review of surface ECG showed two different PVC origins. One of the ECGs indicated PVC origin at LVOT RCC area with inferior axis, left bundle branch block QRS morphology, V3 QRS transition, earlier QRS transition at PVC than that at sinus rhythm, positive $\mathrm{QRS}$ vector in lead I, transition ratio at V2 as 1.2, R-wave duration index as

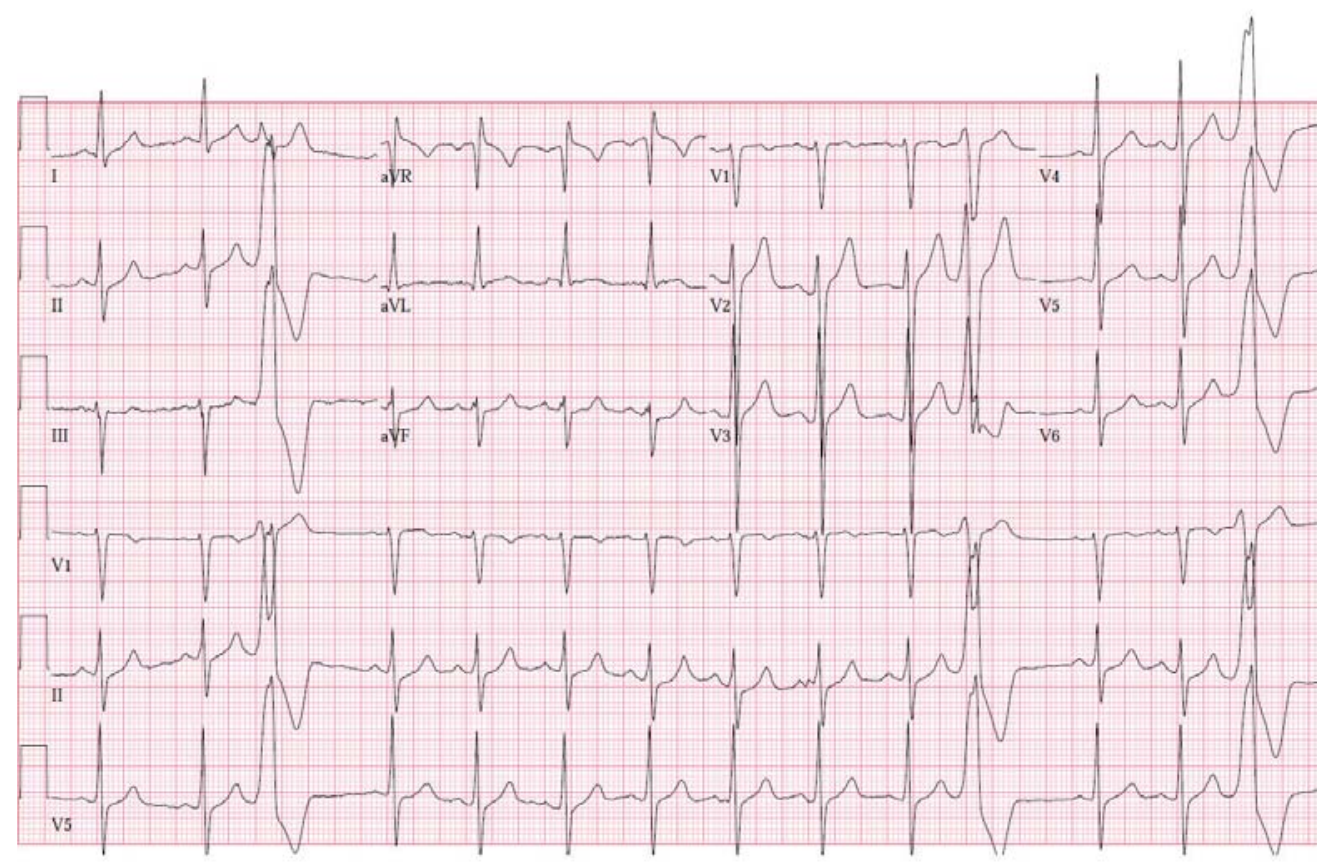

Figure 1. 12-lead Electrocardigram (ECG)

ECG indicated premature ventricular complex (PVC) origin at left ventricular outflow tract right coronary cusp area with inferior axis, left bundle branch block QRS morphology, V3 QRS transition, earlier QRS transition at PVC than that at sinus rhythm, positive QRS vector in lead I, transition ratio at V2 as 1.2, R-wave duration index as 0.5 , and R/S amplitude index as 0.7 . 

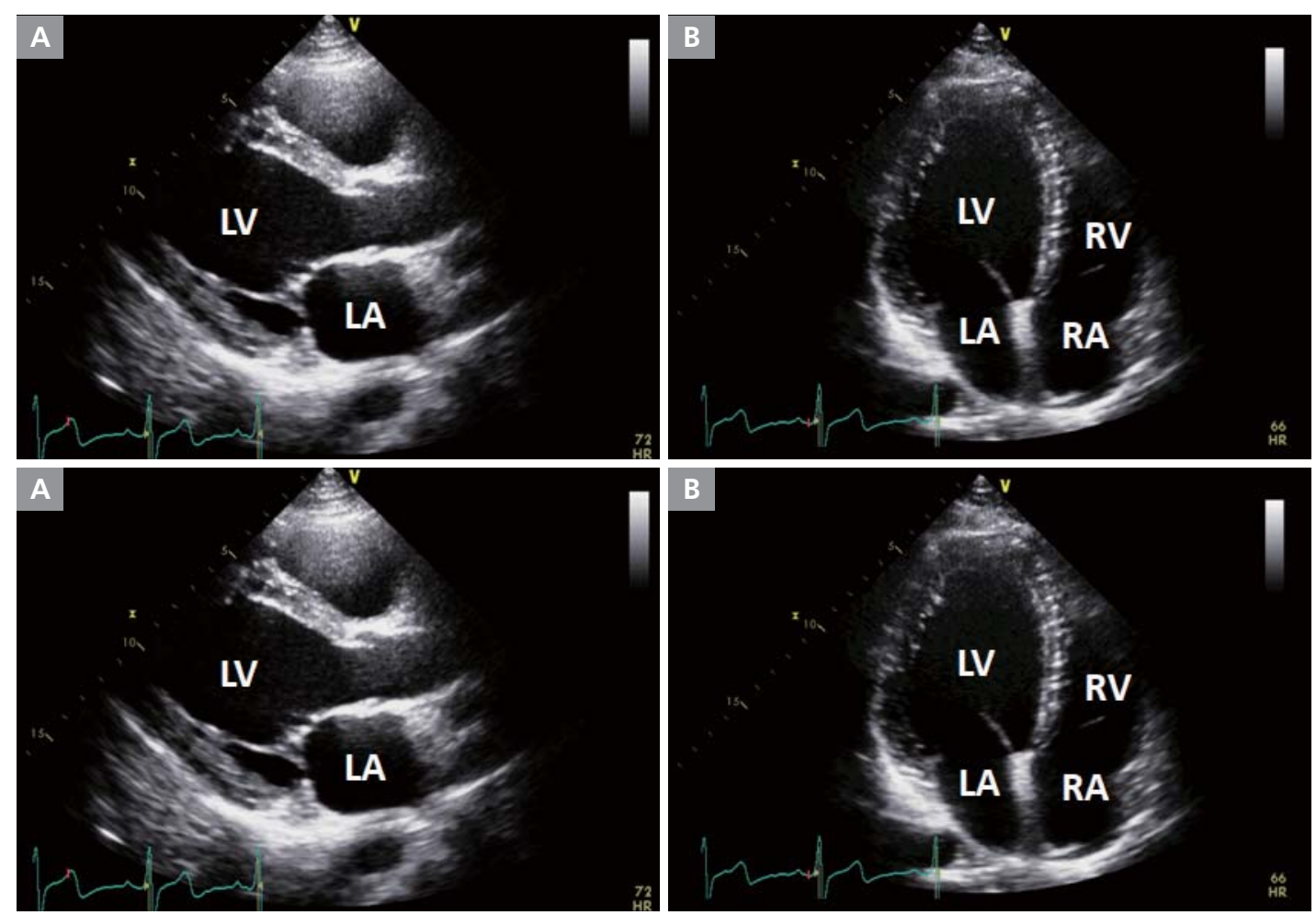

Figure 2. Echocardiogram

(A) parasternal long axis view before ablation (B) apical 4-chamber view before ablation. Left ventricular end-diastolic dimension was dilated to $66 \mathrm{~mm}$ and systolic function was as ejection fraction $40 \%$ by modified Simpson method. (C) parasternal long axis view after ablation (D) apical 4-chamber view before ablation. Left ventricular end-diastolic diastolic dimension was normalized to 55mm as well as left ventricular systolic function was normalized as ejection fraction $55 \%$.

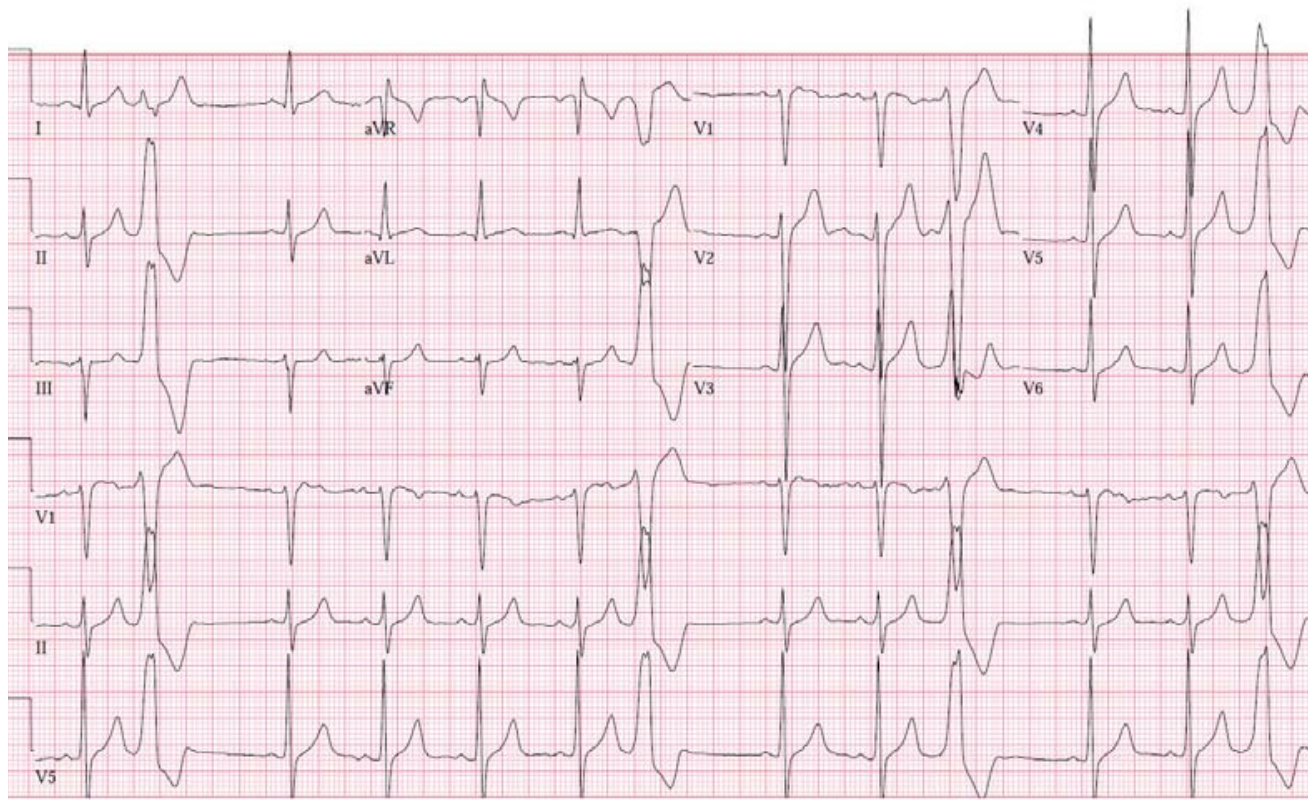

Figure 3. 12-lead Electrocardigram (ECG)

ECG indicated premature ventricular complex (PVC) origin at right ventricular outflow tract (RVOT) posteroseptal area with inferior axis, left bundle branch block QRS morphology, V3 QRS transition, same QRS transition between PVC and sinus rhythm, positive QRS vector in lead I, transition ratio at V2 as 0.9 , R-wave duration index as 0.2 , and R/S amplitude index as 0.2 . 

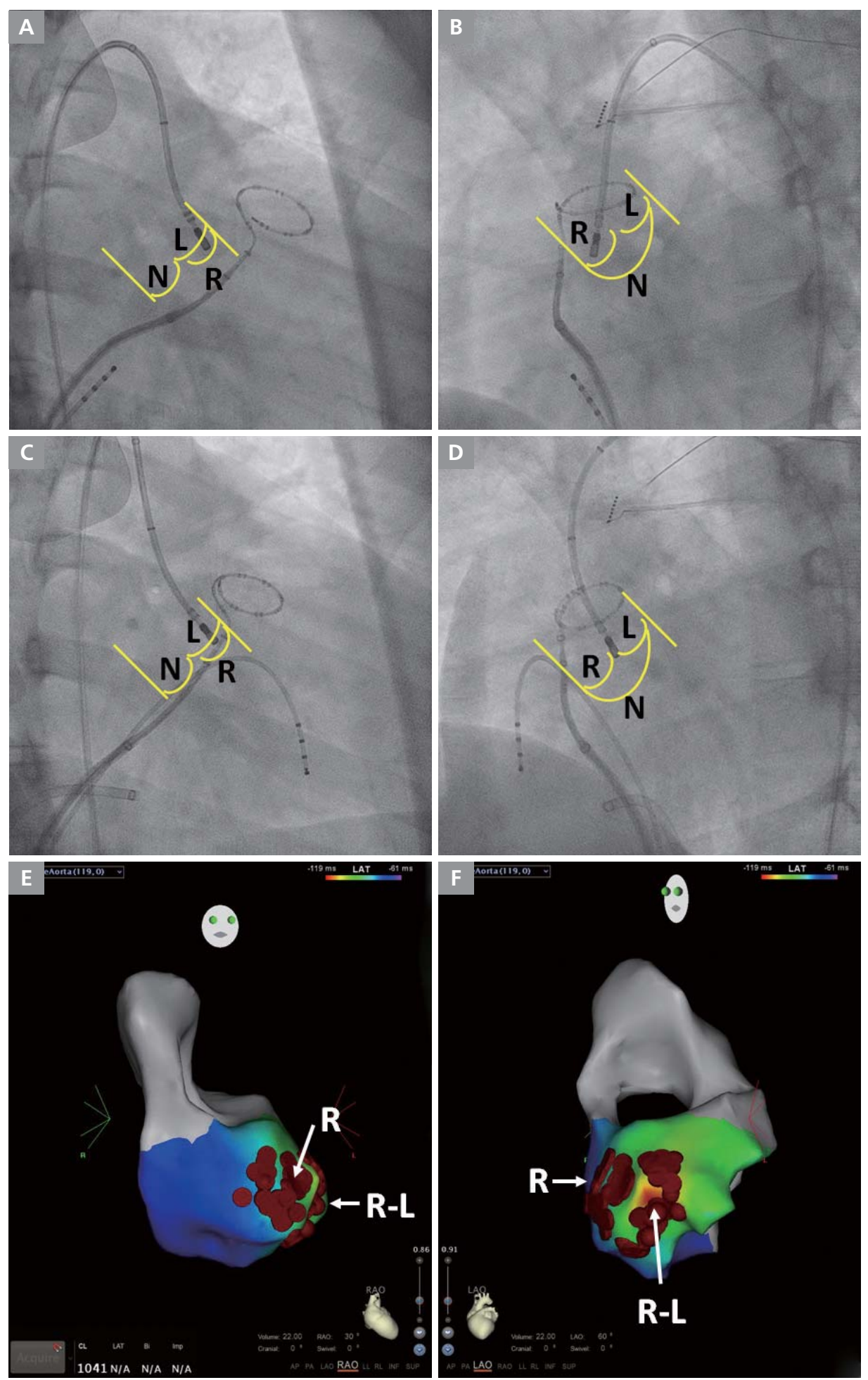

Figure 4. Radiofrequency ablation

(A) Fluoroscopic right anterior oblique view. Ablation catheter is located at left ventricular outflow tract right coronary cusp. (B) Fluoroscopic left anterior oblique view Ablation catheter is located at left ventricular outflow tract right coronary cusp. (C) Fluoroscopic right anterior oblique view. Ablation catheter is located at junction between left ventricular outflow tract right coronary cusp and left coronary cusp. (D) Fluoroscopic left anterior oblique view. Ablation catheter is located at junction between left ventricular outflow tract right coronary cusp and left coronary cusp. (E) Ablation foci (red dot) in 3-dimensional mapping system right anterior oblique view. (F) Ablation foci (red dot) in 3-dimensional mapping system left anterior oblique view. $L=$ left coronary cusp; $N=$ non=coronary cusp; $R$ = Right coronary cusp; $R-L=$ junction between right coronary cusp and left coronary cusp. 


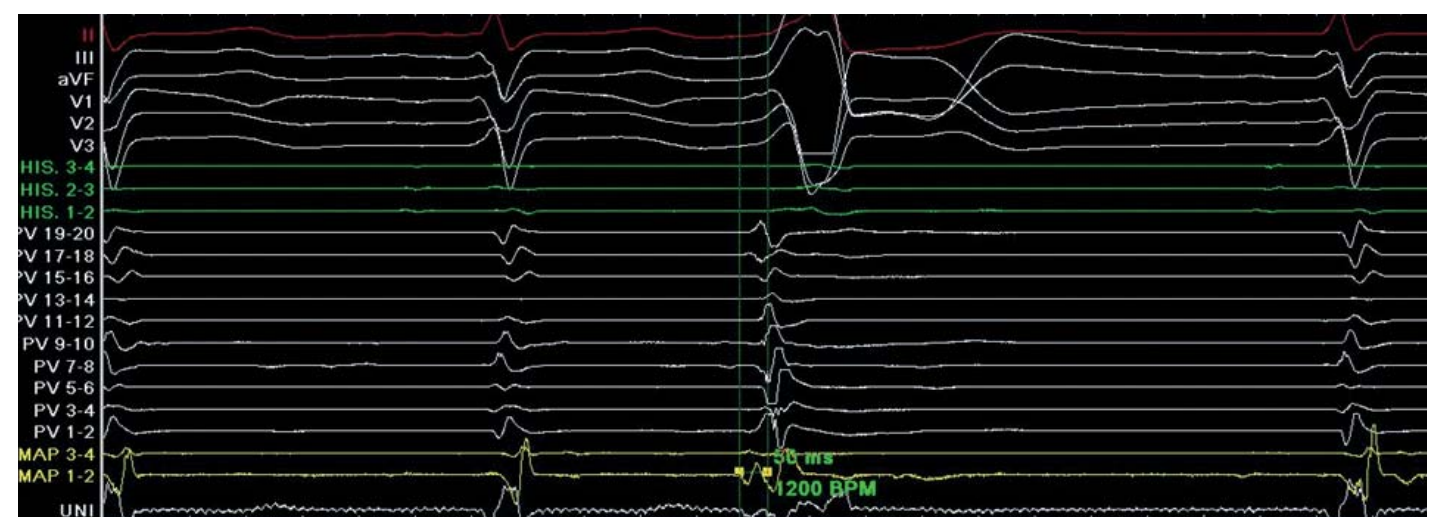

Figure 5. Electrophysiologic study

Earliest premature ventricular complex was mapped at area between left ventricular outflow tract right coronary cusp and left coronary cusp with $50 \mathrm{~ms}$ earlier activation at mapping catheter than surface electrocardiogram.

0.5 , and R/S amplitude index as 0.7 (Figure 1). Another ECG indicated PVC origin at right ventricular outflow tract (RVOT) posteroseptal area with inferior axis, left bundle branch block QRS morphology, V3 QRS transition, same QRS transition between PVC and sinus rhythm, positive QRS vector in lead I, transition ratio at V2 as 0.9, R-wave duration index as 0.2 , and $\mathrm{R} / \mathrm{S}$ amplitude index as 0.2 (Figure 3 ). With the aid of 3-dimensional mapping system (CARTO3 ${ }^{\circ}$, Biosense Webster), electrophysiologic study and detailed mapping were performed. Programmed ventricular extrastimuli at right ventricle apex repetitively induced monomorphic non-sustained ventricular tachycardia. Mapping was sequentially performed at ROVT and LVOT. Earliest PVC was mapped near LVOT RCC with $30 \mathrm{~ms}$ earlier activation at the mapping catheter than the surface ECG. The next PVC was mapped near RVOT posterior septum with $20 \mathrm{~ms}$ earlier activation at the mapping catheter than the surface ECG. At first, radiofrequency ablation was performed at the RVOT posteroseptal area. After that, PVC disappeared, but recurred 5 minutes later. Then, ablation at LVOT was planned. Before ablation, aortogram and coronary angiogram were performed to study the exact anatomy and avoid coronary artery injury. Detailed mapping demonstrated that the earliest PVC was located near the LVOT RCC. Initial ablation at LVOT RCC was successful, but PVC recurred 15 minutes later (Figure 4 A, B). Further mapping demonstrated the earliest PVC to be located in the area between the RCC and the left coronary cusp (LCC) with $50 \mathrm{~ms}$ earlier activation at the mapping catheter than the surface ECG (Figure 5). Within 3 seconds of the first delivery of ablation,
PVC was abolished (Figure 4 C, D). Then, booster ablation was delivered around the initial successful area. After 30 minutes rest, there was no apparent PVC. Finally, ventricular extrastimuli did not induce any PVC or ventricular tachycardia before and after isoproterenol infusion at a rate of $5 \mathrm{ug} / \mathrm{min}$. The patient was discharged with only beta-blocker prescription. Three months later, the patient recovered from disabling palpitations, dyspnea and chest discomfort. All medical treatment was then discontinued. Follow-up Holter examination 6 months postdischarge revealed no PVC, and echocardiogram demonstrated recovery to normal ventricular systolic function and dimensions (Figure 2 C, D).

\section{Discussion}

Medical treatment for PVC in patients with structurally normal hearts is usually successful in suppression of symptoms, and deterioration of ventricular function seldom occurs. However, high burden of PVC might be refractory to anti-arrhythmic drugs, and sometimes results in progression into PVC-mediated dilated cardiomyopathy. ${ }^{1,2}$ In such cases, catheter ablation is a good alternative treatment option, and should be considered before further deterioration of cardiomyopathy.-5 In the present case, the patient showed a structurally normal heart at first presentation, but gradually progressed into DCMP due to high burden of PVC, despite intensive medical therapy. Catheter ablation successfully abolished PVC, and the patient recovered completely with restoration to a structurally normal heart, at present not on 
any anti-arrhythmics.

Medical treatment included beta-blockers, flecainide and propafenone. However, the PVC burden did not decrease and systolic function deteriorated, with an enlarged left ventricular chamber size. Change of anti-arrhythmic drugs to beta-blockers and amiodarone also failed to suppress PVC. Since the patient was complaining of persisted palpitations, chest discomfort and exercise limitation due to dyspnea, catheter ablation was planned according to the current guidelines. ${ }^{3}$

Careful review of 12-lead ECG indicated PVC origin near the LVOT RCC cusp. There was another ECG indicating PVC origin near the RVOT posteroseptal area. These ECG shared common features of outflow tract PVC., 5 There is a possibility that PVC origin shown in both ECGs might have been the same, as posteroseptal aspect of the RVOT is adjacent to the region of LCC in the LVOT, ${ }^{6}$ and precordial lead attachment varies between examiners during each study. The key method to differentiate between RVOT and LVOT origin is precordial QRS transition. ${ }^{7}$ An earlier transition indicates PVC origin from LVOT, whereas a later transition indicates PVC origin from RVOT. However, it is difficult to designate exact location by PVC transition in lead V3, as seen in the present case. In such cases, transition ratio at V2 and R/S index are useful. ${ }^{8.9} \mathrm{PVC}$ origin from LVOT has transition ratio at V2 more than $0.6,{ }^{8} \mathrm{R}$-wave duration index at V1/V2 and V4 more than 0.3, and R/S amplitude index at $\mathrm{V} 1$ or $\mathrm{V} 2$ more than $0.5 .^{9}$ In the present case, transition ratio at V2 was 1.2 and 0.7; both indicated PVC origin from LVOT. However, R-wave duration index and R/S amplitude index indicated PVC origin from both RVOT and LVOT. Therefore, mapping was performed both at RVOT and LVOT.

Before catheter ablation, angiography at both outflow tracts and coronary arteries were performed, to delineate accurate anatomy and avoid unnecessary damage. Initial ablation attempts at RVOT seemed successful. However, they failed to eliminate PVC. Initial disappearance of PVC might be due to partial thermal injury to the exact PVC origin near the LVOT RCC, adjacent to the ablation area at the RVOT posteroseptum. Serial ablation at the LVOT RCC and junction between the RCC and LCC, which was indicated by surface ECG and 3-dimensional mapping, successfully eliminated PVC.

After discharge, the patient did not complain of palpitations and dyspnea any more, even without anti-arrhythmic drugs. Follow-up Holter examination demonstrated no PVC as well as recovery to a structurally normal heart on echocardiogram.

High burden of PVC refractory to medical therapy can lead to deterioration of cardiac function. Not all cases of DCMP are caused by PVC or ventricular tachycardia. However, PVCinduced cardiomyopathy is a reversible condition with high chances of recovery to normal systolic function and chamber size. ${ }^{4,10}$ Persistent high burden of PVC causes severe deterioration of cardiac function. In this situation, catheter ablation might be curative rather than palliative. Therefore, catheter ablation should be promptly applied when PVC promotes ventricular dysfunction refractory to medical therapy.

\section{Reference}

1) Lee AK, Deyell MW. Premature ventricular contraction-induced cardiomyopathy. Curr opin Cardiol. 2016;31:1-10.

2) Penela D, Fernandez-Armenta J, Aguinaga L, Tercedor L, Ordonez A, Bisbal F, Acosta J, Rossi L, Borras R, Doltra A, Ortiz-Perez JT, Bosch X, Perea RJ, Prat-Gonzalez S, Soto-Iglesias D, Tolosana JM, Vassanelli F, Cabrera M, Linhart M, Martinez M, Mont L, Berruezo A. Clinical recognition of pure premature ventricular complex-induced cardiomyopathy at presentation. Heart Rhythm. 2017;14:1864-1870.

3) Priori SG, Blomstrom-Lundqvist C, Mazzanti A, Blom N, Borggrefe M, Camm J, Elliott PM, Fitzsimons D, Hatala R, Hindricks G, Kirchhof P, Kjeldsen K, Kuck KH, HernandezMadrid A, Nikolaou N, Norekval TM, Spaulding C, Van Veldhuisen DJ. 2015 ESC guidelines for the management of patients with ventricular arrhythmias and the prevention of sudden cardiac death: the task force for the management of patients with ventricular arrhythmias and the prevention of sudden cardiac death of the European Society of Cardiology (ESC). Endorsed by: Association for European Paediatric and Congenital Cardiology (AEPC). Eur Heart J. 2015;36:2793-2867.

4) Baman TS, Lange DC, Ilg KJ, Gupta SK, Liu TY, Alguire C, Armstrong W, Good E, Chugh A, Jongnarangsin K, Pelosi F, Jr., Crawford T, Ebinger M, Oral H, Morady F, Bogun F. Relationship between burden of premature ventricular complexes and left ventricular function. Heart Rhythm. 2010;7:865-869.

5) Tanawuttiwat $T$, Nazarian $S$, Calkins $H$. The role of catheter 
ablation in the management of ventricular tachycardia. Eur Heart J. 2016;37:594-609.

6) Ho SY. Anatomic insights for catheter ablation of ventricular tachycardia. Heart Rhythm. 2009;6:S77-80.

7) Hutchinson MD, Garcia FC. An organized approach to the localization, mapping, and ablation of outflow tract ventricular arrhythmias. J Cardiovasc Electrophysiol. 2013;24:1189-1197.

8) Betensky BP, Park RE, Marchlinski FE, Hutchinson MD, Garcia FC, Dixit S, Callans DJ, Cooper JM, Bala R, Lin D, Riley MP, Gerstenfeld EP. The $\mathrm{V}(2)$ transition ratio: A new electrocardiographic criterion for distinguishing left from right ventricular outflow tract tachycardia origin. J Am Coll Cardiol. 2011;57:2255-2262.
9) Ouyang F, Fotuhi P, Ho SY, Hebe J, Volkmer M, Goya M, Burns M, Antz M, Ernst S, Cappato R, Kuck KH. Repetitive monomorphic ventricular tachycardia originating from the aortic sinus cusp: Electrocardiographic characterization for guiding catheter ablation. J Am Coll Cardiol. 2002;39:500-508.

10) Takemoto M, Yoshimura H, Ohba Y, Matsumoto Y, Yamamoto U, Mohri M, Yamamoto H, Origuchi H. Radiofrequency catheter ablation of premature ventricular complexes from right ventricular outflow tract improves left ventricular dilation and clinical status in patients without structural heart disease. J Am Coll Cardiol. 2005;45:1259-1265. 